\title{
A support programme for registered nurses in the early identification of autism spectrum disorders in primary healthcare clinics: A pilot
} study

A-M Wium, DPhil (Commun Pathol); M de Jongh, $\mathrm{PhD}$ (Psychol)

Department of Speech-Language Pathology and Audiology, School of Health Sciences, Sefako Makgatho Health Sciences University, Ga-Rankuwa, South Africa

Corresponding author: $M$ de Jongh (marguerite.dejongh@smu.ac.za)

\begin{abstract}
Background. Registered nurses in South Africa often work in community clinics in primary healthcare (PHC), where they are first in line to be consulted by families with children with disabilities. There has been limited research with a focus on interprofessional collaboration regarding autism spectrum disorders (ASDs) in the local context. A support programme was developed to inform registered nurses in PHC of the symptoms and characteristics of ASDs in order to increase the number of referrals to specialists for early diagnosis.

Objectives. To determine the effectiveness of a support programme for registered nurses in PHC clinics, and to raise awareness of ASDs.

Methods. Two PHC clinics in a semirural area in Gauteng Province, SA, were included in the study, in which 10 registered nurses participated. A descriptive, quantitative approach was used, and data were collected by means of self-administered questionnaires.

Results. The results confirmed that the support was effective, as participants showed an increase in knowledge and understanding of ASDs. Poor attendance and the limited sample size affected the outcomes.

Conclusion. A multidisciplinary approach to the early identification and referral of children with possible ASDs is important to improve the quality of life of these children and prevent further delays. Speech-language therapists should provide support to registered nurses in PHC, and training should be repeated on a continual basis to facilitate long-term retention and to accommodate shift changes within clinics.
\end{abstract}

Afr J Health Professions Educ 2018;10(2):136-140. DOI:10.7196/AJHPE.2018.v10i2.963

Autism spectrum disorders (ASDs) encompass a wide spectrum of symptoms and levels of impairment in the social, communicative and behavioural domains. ${ }^{[1]}$ There has been limited research with a focus on interprofessional collaboration regarding ASDs in the local context. The intention of the present research was to develop a support programme that would allow registered nurses in primary healthcare (PHC) to identify the characteristics of children with ASDs and to recognise possible risk factors. This practice would allow nurses to refer such children to appropriate healthcare services, and to provide parents with essential information when there is reason for concern.

PHC is the first level of contact between the general population and the health system. ${ }^{[2]}$ Patients are referred to healthcare services at secondary and tertiary hospitals. ${ }^{[3]}$ Nurses in PHC often have to consult with the families of children with disabilities. ${ }^{[4]}$ As registered nurses interact with mothers about the welfare of their children, and become aware of their concerns, they require unique knowledge, competencies and skills. ${ }^{[3]}$ It is therefore important that nurses in PHC are made aware of the characteristics and risk factors associated with ASDs (e.g. social, communicative and behavioural problems, and learning characteristics) in order for them to refer children as soon as possible. ${ }^{[5]}$

Early diagnosis provides an opportunity for early intervention, which improves the prognosis of the child in terms of linguistic, cognitive, social and motor abilities. ${ }^{[6]}$ In rural contexts, ASDs are often only diagnosed many years after the onset of symptoms, or misdiagnosed, possibly as a result of a lack of knowledge. ${ }^{[7]}$
Awareness and knowledge of autism is very limited among healthcare workers in sub-Saharan African countries; ${ }^{[6]}$ early diagnosis and intervention occur more often in developed countries. ${ }^{[8]}$ Because of this situation, the diagnosis and detection of ASDs often occurs too late. Valuable time is lost in which intervention and education could have occurred. In some African countries, the proportion of children with ASDs receiving an education can be as low as $1-3 \% \cdot{ }^{[9]}$ Early identification is crucial for the development, education and functioning of the child, and should occur before the age of $3 .{ }^{[10]}$

Registered nurses in PHC clinics spend most of their time treating and diagnosing common childhood diseases (e.g. diarrhoea, nutritional deficits, HIV/AIDS) that are related to the national burdens of disease, and are less focused on behavioural difficulties in young children. ${ }^{[3]}$ In addition, registered nurses receive limited information on the topic of ASDs in their professional training. Lack of knowledge about ASDs presents barriers to early identification of and intervention for such children. ${ }^{[5]}$ Previous research has shown that there is a critical need to train healthcare workers in recognising ASDs, especially in underserved communities. ${ }^{[7,11]}$

Early intervention can significantly improve the quality of life of children with $\mathrm{ASDs}^{[3]}$ and prevent further delays. ${ }^{[10]}$ Registered nurses should be supported in order to become familiar with the characteristics and risk factors associated with ASDs, and to understand the impact of ASDs on children and their families. Signs of ASDs often appear at the age of $12-18$ months ${ }^{[12]}$ Children are usually seen at clinics at regular intervals $(6,12,18$, 24 and 30 months) for immunisation, and on such occasions parents may want to discuss their concerns with the nurse. It is important that registered 
nurses at clinics are aware of the characteristics of ASDs and the possible risk factors, so that they can refer a child with suspected ASD to a specialist physician for early diagnosis and intervention. ${ }^{[12,13]}$

A comprehensive diagnostic evaluation of ASDs involves a multidisciplinary team comprised of a paediatric nurse, psychiatrist, psychologist, neurologist, speech-language therapist and occupational therapist. ${ }^{[14]}$ In reality, many children with ASD are diagnosed too late to obtain optimum benefit from early intervention services. It is estimated that $<50 \%$ of children with ASDs are diagnosed before the age of $3-6$ years, and that black children are diagnosed much later than white children. ${ }^{[7]}$ This might be a result of inadequate screening practices, slow responses to parental concerns, or a failure to recognise symptoms early in life. The findings suggest that there may be differences in recognition of symptoms and diagnosis across racial and ethnic groups. It may also be a result of differences in accessing information about ASDs and lack of continuity in the provision of healthcare services. In the South African (SA) context, these differences might be attributable to geography rather than simply race, because of a history of racial segregation. The Legislative Blue Ribbon Commission on Autism revealed that in general, children with ASDs might also experience a 13-month delay between the initial evaluation and actual diagnosis. ${ }^{[15]}$

When parents of a child with ASD consult a registered nurse at a PHC clinic, they expect answers to their questions on how to manage their child. Suitable guidance would allow both the family and the child the best quality of life. ${ }^{[14]}$ Early intervention through the use of screening, psychological support and education on ASDs can enable the family to cope and adjust to the necessary changes related to the child's diagnosis. ${ }^{[16]}$ The present study focused on training registered nurses in PHC clinics in a semirural context on ASD in children.

\section{Methods}

Two PHC clinics in a semirural area in Gauteng Province, SA, were included in the study, and 10 registered nurses participated. In this specific context, referrals are made from primary to tertiary level, as there are no secondary hospitals in close proximity to the clinics.

The aim of the study was to determine the effectiveness of the support provided in terms of (i) the training process, and (ii) the knowledge gained about ASDs.

The study was designed as intervention research (Fig. 1), using a quantitative approach with a pretest post-test design in the evaluation phase. A questionnaire designed for the study purposes was used, which consisted of mostly closed-ended questions, expanded on by a limited number of open-ended questions. The pre-test questionnaire also included questions about demographic information. The questionnaires collected information on the participants' knowledge of ASDs pre- and post-training, to determine the effectiveness of the support provided. The workshops were evaluated on a rating scale from 1 - 4. The content and clarity of the questions as well as the face validity in the questionnaires was confirmed prior to the study by obtaining the opinions of three volunteers in the nursing department who were experts in the field of PHC.

A pilot study was conducted with three registered nurses at a clinic that was not included in the study, to test the training and instructions. Bias was minimised by not asking sensitive questions of a personal nature, and numbering the questionnaires to ensure anonymity. The questions were based on findings from the literature review and the objectives of the study.

Ethical clearance for the research was obtained from Sefako Makgatho Health Sciences University's Research and Ethics Committee (ref. no. MREC/H/78/2012: UG), and the Gauteng Department of Health. All ethical principles were adhered to in the design of the study.

The researchers conducted a briefing session at the two clinics to explain the rationale for the study and what would be expected from the participants, before obtaining informed consent. Arrangements were made for the training sessions. The Gauteng Department of Health determined the particular dates for training in accordance with their continuing professional development schedule. The workshops were designed according to adult learning principles, and were facilitated by the researchers.

The participants were registered nurses with a 4-year qualification, who had not obtained any additional specialised qualification (e.g. in psychiatry or paediatrics). The two PHC clinics were purposively selected as they were at a reasonable distance from the tertiary institution.

The two training sessions of 1 hour each were scheduled over 2 consecutive weeks at each of the clinics. The first session focused on defining and describing the characteristics, prevalence and causes of ASDs, as well as associated risk factors. The second addressed the role of registered nurses in the identification of ASDs, parent education and the available resources for children with ASDs.

Although a large number of registered nurses $(n=21)$ initially provided informed consent to

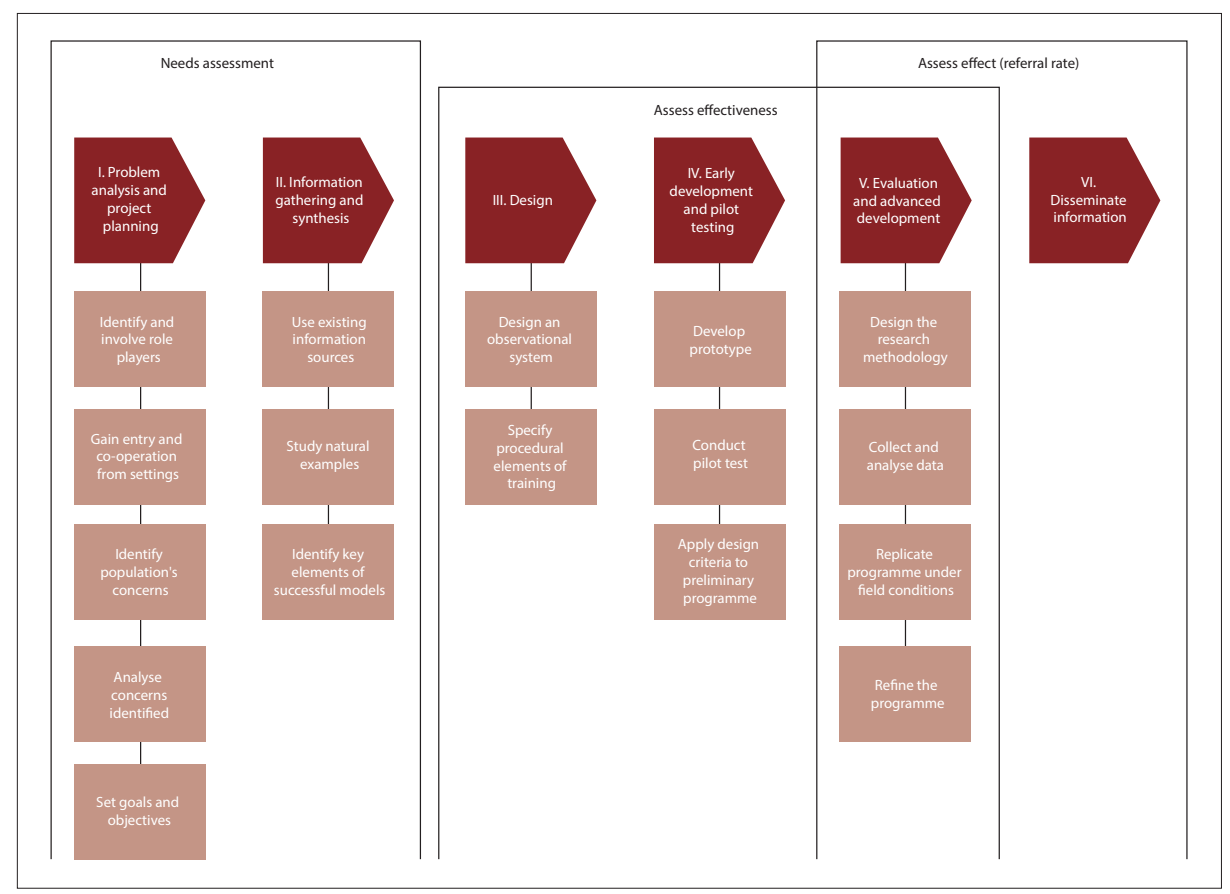

Fig. 1. Framework for intervention research. ${ }^{[17]}$ 
participate in the research at the initial briefing sessions and attended at least one of the workshops, only those who attended both workshops and completed both the questionnaires were included in the research $(N=10)$. The high attrition rate (52\%) was due to shift changes and availability on the prearranged days of the workshops.

The researchers, who were independent from the clinics, distributed and collected the questionnaires by hand. Baseline knowledge had been determined by completing the questionnaire prior to the workshops, and was then compared with the post-training results to obtain knowledge gains. Following each workshop, the participants rated the presentation. The quantitative data were entered into an Excel (Microsoft, USA) spreadsheet to be analysed descriptively. Results are presented as percentages (Table 1). Qualitative answers were listed in a table format and quantified on a threepoint scale as either correct (2), partially correct (1), or incorrect (0), according to a memorandum. Knowledge gains were based on a comparison between pre-and post-training scores.

\section{Results}

The effectiveness of the support provided was determined by participants' ratings of the length of the presentation, the language used, the preparedness of the presenters, the style and format of the presentations and the value of the support provided. The majority (90\%) regarded the length of the workshops ( 1 hour) as being adequate. All the participants $(N=10)$ viewed the language used to conduct the workshops as adequate and easy to understand. The presentations were conducted in an explanatory manner in English, which is the language of higher education and training in SA. ${ }^{[20]}$ All the participants $(N=10)$ considered the researchers to have been adequately prepared for the workshops. The style and format of the presentations were considered to be 'interesting' by all the participants. The workshop format, using PowerPoint (Microsoft, USA) together with discussions, appealed to the participants.

These results confirm that the presentations and information were valued and interesting, because the content was new but relevant to the participants. The participants also asked several questions and made comments that elicited discussion during the presentations.

Fig. 2 shows participants' perceptions of benefits obtained from the training.

Of those participants who gained (100\%) in knowledge, $90 \%$ indicated that they gained significantly. These results were confirmed by comparing the pre- and post-training questionnaires (Table 1). This comparison showed definite gains in knowledge, as only 50\% $(n=5)$ of participants could explain the concept of ASD to some extent prior to training, while all $(100 \%)$ could do so after training. There is, however, room for improvement and a need for further training, as $70 \%$ could still only partially explain what ASD entails (Table 2) ${ }^{[13]}$ following training. Such results imply an emergent understanding of the concept. The ideal would be to provide the participants with additional support, to generate a full understanding of the concept by all the participants.

There appears to have been an increase in the level of understanding of specific problems associated with autism, as $90 \%$ of the participants had only limited knowledge pre-training (partially correct or incorrect responses), in contrast to $50 \%$ post-training, though this still leaves room for improvement. Similar knowledge gains can be seen in participants' understanding of the age at which autism appears, as only $50 \%$ of the participants described it correctly prior to the support provided, in contrast to $90 \%$ after the training. ASDs appear during the first 36 months of life, ${ }^{[5]}$

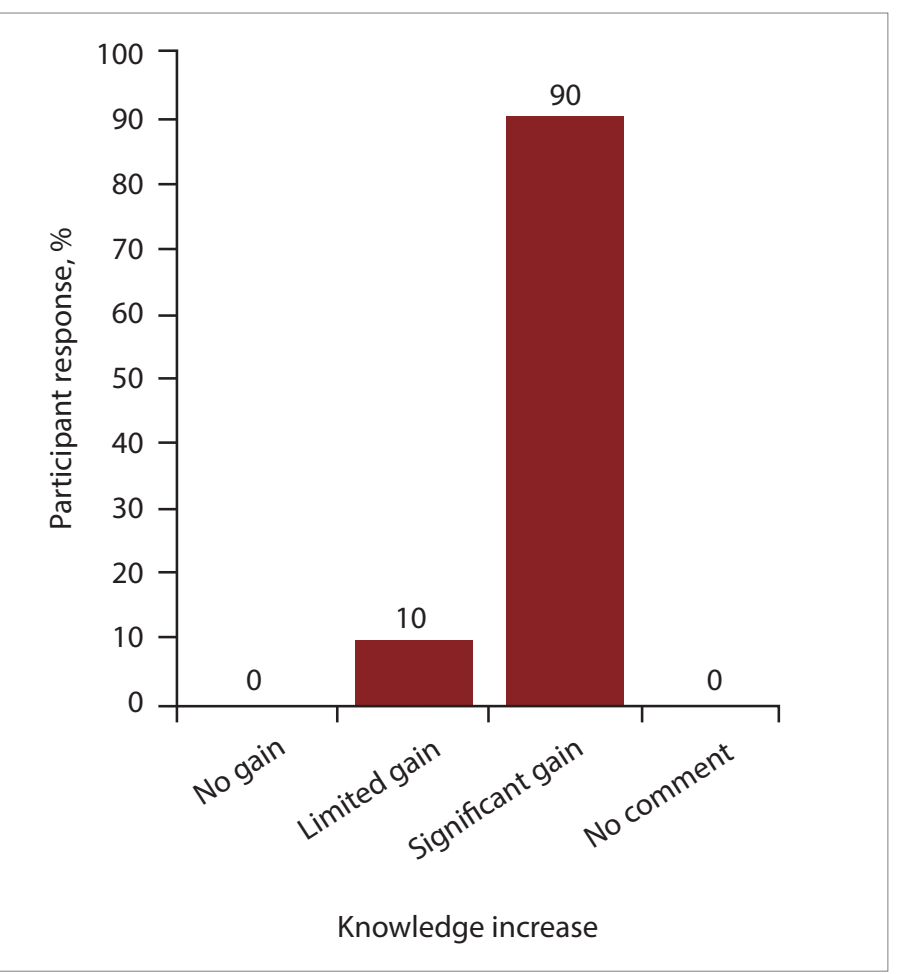

Fig. 2. Participant rating of benefits of the presentation $(\mathrm{N}=10)$.

Table 1. Comparison of pre- and post-training knowledge/understanding of autism spectrum disorders $(N=10)$

\begin{tabular}{|c|c|c|c|c|c|c|}
\hline & \multicolumn{3}{|c|}{ Pre-test questionnaire answers, $\%$} & \multicolumn{3}{|c|}{ Post-test questionnaire answers, $\%$} \\
\hline & & Partially & & & Partiall & \\
\hline & Correct & correct & Incorrect & Correct & correct & Incorrect \\
\hline What do you consider autism to be? & 0 & 50 & 50 & 30 & 70 & 0 \\
\hline Autism is characterised by specific problems. Please list. & 10 & 50 & 40 & 50 & 40 & 10 \\
\hline At what age does autism usually appear? & 40 & 10 & 50 & 90 & 0 & 10 \\
\hline What are the educational alternatives/options for a child with autism? & 40 & 0 & 60 & 50 & 0 & 50 \\
\hline Which other problems can be associated with autism? & 20 & 20 & 60 & 60 & 30 & 10 \\
\hline Is autism hereditary? & 40 & 30 & 30 & 50 & 20 & 30 \\
\hline Who is responsible for the diagnosis of autism? & 10 & 70 & 20 & 40 & 50 & 10 \\
\hline What are the causes of autism? & 30 & 20 & 50 & 70 & 30 & 0 \\
\hline Where do you refer a child when you suspect an autism spectrum disorder? & 0 & 80 & 20 & 90 & 0 & 10 \\
\hline What are the treatment approaches for a child with an autism spectrum disorder? & 10 & 20 & 70 & 50 & 10 & 40 \\
\hline
\end{tabular}


Table 2. Characteristics of autism spectrum disorders ${ }^{[12]}$

\begin{tabular}{|c|c|c|c|}
\hline Social characteristics & Communication characteristics & Behavioural problems & Learning characteristics \\
\hline $\begin{array}{l}\text { Poor pretend play skills } \\
\text { Poor eye contact } \\
\text { Inappropriate display of emotions } \\
\text { Limited interest in social interaction } \\
\text { Inability to understand gestures, } \\
\text { social cues and facial expressions }\end{array}$ & $\begin{array}{l}\text { Difficulty reading and expressing } \\
\text { emotions } \\
\text { Inability to babble or coo } \\
\text { Language delay } \\
\text { Echolalia } \\
\text { Limited imitation } \\
\text { Difficulty using gestures and facial } \\
\text { expression and understanding social } \\
\text { language } \\
\text { Limited vocabulary } \\
\text { Inadequate use of grammatical } \\
\text { structures, intonation, pitch, rhythm } \\
\text { and stress } \\
\text { Short attention span } \\
\text { Disorganised }\end{array}$ & $\begin{array}{l}\text { Dislike touch } \\
\text { Do not always respond to auditory } \\
\text { stimuli } \\
\text { Show a lack of fear for danger } \\
\text { Avoid eye contact } \\
\text { Are preoccupied with own thoughts } \\
\text { Are less flexible in adapting to } \\
\text { changing routines } \\
\text { Focus on one activity at a time } \\
\text { Explore the environment } \\
\text { inappropriately, e.g. licking, smelling, } \\
\text { handling of objects } \\
\text { Injure themselves by banging head } \\
\text { against table, etc. }\end{array}$ & $\begin{array}{l}\text { Hypersensitivity or hyposensitivity to } \\
\text { sensory stimuli such as textures, taste, } \\
\text { smells, sounds, visual input, pain } \\
\text { Poor fine motor skills, e.g. difficulty } \\
\text { holding a pencil, drawing or writing, } \\
\text { etc. } \\
\text { Gross motor difficulties, e.g. } \\
\text { coordination and balance problems, } \\
\text { etc. } \\
\text { Easily frustrated }\end{array}$ \\
\hline
\end{tabular}

and it is during this period that parents usually consult PHC clinics and express their concerns. Registered nurses should be vigilant and able to identify the risk factors and symptoms of ASDs, which include social, communicative and learning characteristics, as well as behavioural problems. ${ }^{[5]}$

There was also an increase in the proportion of participants $(30 \%)$ who understood who the professionals were to refer patients to for diagnosis of ASDs. Less improvement (10\%) was seen in their knowledge about the availability of schools and resources for children with ASDs. However, the participants asked the facilitators after the workshops for an information leaflet on this, so that they could make proper referrals. A 50\% increase in knowledge was seen with regards to the conditions comorbid with ASDs (e.g. mental illnesses such as attention deficit hyperactivity disorder and anxiety disorder).

With regards to the causes of ASDs, all participants $(n=10)$ provided either a partially correct $(30 \%)$ or correct $(70 \%)$ answer after the workshops, in contrast to the $50 \%$ of participants who were completely wrong before training.

The majority of participants (90\%) knew which facility to refer children with ASDs to after the training, which shows an increase of $10 \%$. It could therefore be expected that the registered nurses who took part in this training would refer children for diagnosis to the tertiary hospital. ${ }^{[4]}$

A more significant improvement was seen in their knowledge of treatment approaches for ASDs. Prior to training, only $30 \%$ of the registered nurses had some idea of the different treatment approaches, compared to $60 \%$ following the training. These registered nurses had received general training that did not include specialised training in psychiatrics or paediatrics, and they had no prior knowledge of ASDs. It is therefore suggested that registered nurses collaborate with other members of health teams in order to meet the healthcare needs of the public. ${ }^{[11]}$

\section{Discussion}

Based on the overall results, it can be seen that participants' knowledge improved and that they developed a better understanding of the condition. The information leaflet that we provided upon request, with contact numbers of appropriate schools, was considered helpful in advising parents on educational options.

These workshops raised awareness of ASDs among the participants, but there remains a need for added support. Registered nurses in PHC clinics are not expected to diagnose ASDs, but they should be made aware of the risk factors and associated characteristics. They should additionally be able to refer parents to specialists and schools for learners with special educational needs. Support should ideally be provided on a continual basis, to refresh their knowledge and to accommodate colleagues who could not attend the original presentations. It was found to be valuable to have discussion groups following training, because the nurses showed a need to discuss the relevant issues pertaining to the topic.

The limitations of the study were the low attendance and small sample size. The low attendance (high attrition rate) of the nurses at the two clinics can be attributed to the nature of their work, as shift schedules complicated this matter. As the specific dates for training were predetermined by the Gauteng Department of Health for staff development purposes, no flexibility was possible, and so we could not accommodate all the registered nurses.

In addition, this project allowed only two clinics to be included in the research. Future support programmes should be conducted in all clinics in the catchment area of the hospital, before an increased referral rate to specialists for early diagnosis of ASDs is likely to be seen. The results of this study should be interpreted with regards to the limited sample size. Purposive sampling further limits the generalisation of these results to other contexts.

\section{Conclusion}

The programme was presented in an effective manner, and participants gained knowledge about ASDs. This condition remains a challenge, owing to the global increase in its prevalence. ${ }^{[20]}$ ASDs should be addressed in nurses' professional training, so that they can identify behaviour, symptoms and risk factors, in order to refer these children for diagnosis at an early age, which will improve the prognosis of the condition.

A multidisciplinary approach where speechlanguage therapists (SLTs) and registered nurses work together in the early identification and referral of children with possible ASDs is needed. In accordance with their scope of practice, SLTs should provide support to registered nurses in PHC to enhance their knowledge of ASDs. Effective support by SLTs should be based on interprofessional teamwork, and on encouraging adequate attendance of workshops. However, shift schedules are not necessarily flexible, and it may be necessary to repeat workshops to accommodate more registered nurses. The scheduling of 
workshops for continuing professional development is an administrative matter that needs to be negotiated with the Department of Health.

Acknowledgements. We wish to acknowlege the following students for the data collection: Caley-Jade Selepe, Duduzile Nhlapo, Glen Shivambu and Kgaugelo Tema.

Author contributions. Equal contributions.

Funding. None.

\section{Conflicts of interest. None.}

Newschaffer CJ, Croen LA, Daniels J, et al. The epidemiology of autism spectrum disorders. Annu Rev Public Health 2007;28(1):235-258. https://doi.org/10.1146/annurev.publhealth.28.021406.144007

2. Thema LK, Singh S. Integrated primary oral health services in South Africa: The role of the PHC nurse in providing oral health examination and education. Afr J Prim Health Care Fam Med 2013;5(1):1-4. https://doi. org/10.4102/phcfm.v5i1.413

3. Karande S. Autism: A review for family physicians. Indian J Med Sci 2006;60(5):205-215. https://doi. org/10.4103/0019-5359.25683

4. Bonis S. Stress and parents of children with autism: A review of the literature. Issues Ment Health Nurs 2016;37(3):153-163. https://doi.org/10.3109/01612840.2015.1116030

5. Boyd BA, Odom SL, Humphreys BP, Sam AM. Infants and toddlers with autism spectrum disorder: Early identification and early intervention. J Early Interv 20101;32(2):75-98. https://do org/10.1177\%

6. Bakare MO, Ebigbo PO, Agomoh AO, et al. Knowledge about childhood autism and opinion among healthcar workers on availability of facilities and law caring for the needs and rights of children with childhood autism an other developmental disorders in Nigeria. BMC Pediatr 2009;9(1):12. https://doi.org/10.1186/1471-2431-9-12
7. Mandell DS, Wiggins LD, Carpenter LA, et al. Racial/ethnic disparities in the identification of children with autism spectrum disorders. Am J Public Health 2009:99(3):493-498. https///doi.org/10.2105/AJPH.2007.131243 8. Bakare MO, Munir KM. Autism spectrum disorders (ASD) in Africa: A perspective. Afr J Psychiatry 2011;14(3):208-210. https://doi.org/10.4314/aipsy.v14i3.3

9. Bowker A, D’Angelo NM, Hicks R, Wells K. Treatments for autism: Parental choices and perceptions of change. J Autism Dev Disord 2011;41(10):1373-1382. https://doi.org/10.1007/s10803-010-1164-y

10. Beukelman D, Mirenda P. Augmentative and alternative communication: Supporting children and adults with complex communication needs. Baltimore: Paul Brooks, 2012.

11. Van der Linde J, Kritzinger A. Perceptions of rural primary healthcare personnel about expansion of early communication intervention: Original research. Afr J Prim Health Care Fam Med 2013;5(1):1-11. https://dol org/10.4102\%2Fphcfm.v5i1.553

12. Weissman L, Bridgemohan C. Autism spectrum disorder in children and adolescents: Overview of management. UpToDate, 2018. https://www.uptodate.com/contents/autism-spectrum-disorder-in-children-and-adolescentsUpToDate, 2018. https://www.uptodate.com/cont
overview-of-management (accessed 13 May 2018).

13. Christensen DL, Bilder DA, Zahorodny W, et al. Prevalence and characteristics of autism spectrum disorder among 4-year-old children in the autism and developmental disabilities monitoring network. J Dev Behav Pediats among 4-year-old children in the autism and developmental disa

14. Elder JH, D’Alessandro T. Supporting families of children with autism spectrum disorders: Questions parents ask and what nurses need to know. Pediatr Nurs 2009;35(4):240.

5. Henderson K. Policies and practices used by states to serve children with autism spectrum disorders. J Disabi Policy Stud 2011;22(2):106-115. https://doi.org/10.1177/1044207310396210

6. Hall HR, Graff JC. The relationships among adaptive behaviors of children with autism, family support, parenting stress, and coping. Issues Compr Pediatr Nurs 2011;34(1):4-25. https://doi.org/10.3109/01460862.2011.555270 7. Thomas EJ, Rothman J. Intervention Research: Design and Development for Human Service. Haworth: Boston,

18. Matson JL, Kozlowski AM. The increasing prevalence of autism spectrum disorders. Res Autism Spectr Disord 2011;5(1):418-425. https://doi.org/10.1016/.j.rasd.2010.06.004

Accepted 10 October 2017 